\title{
PERCEPÇÃO DAS MÃES SOBRE O PROGRAMA NACIONAL DE SUPLEMENTAÇÃO DE VITAMINA A NA CIDADE DE CAMPINA GRANDE - PB
}

\author{
PERCEPTION OF MOTHERS ABOUT THE NATIONAL VITAMIN A \\ SUPPLEMENTATION PROGRAM IN THE CITY OF CAMPINA \\ GRANDE - PB
}

\author{
Wanessa de Oliveira Mendonça ${ }^{1}$ \\ Lidiane da Conceição Nóbrega de Araújo ${ }^{2}$ \\ Davyson Barbosa Duarte ${ }^{3}$ \\ Iris Damião Macena ${ }^{4}$ \\ Mayana Kelly Tavares de Souza ${ }^{5}$
}

\begin{abstract}
RESUMO: OBJETIVO: Esta pesquisa teve como objetivo avaliar a percepção das mães e/ou responsáveis acerca do PNVITA. MÉTODO: Trata-se de um estudo exploratório, descritivo, de natureza aplicada. A abordagem foi constituída de pesquisa qualitativa e quantitativa. A pesquisa foi realizada em quatro UBSF do município de Campina Grande - PB. A coleta de dados consistiu na aplicação de um questionário sobre a vitamina A e o PNVITA contendo as variáveis socioeconômicas e demográficas a fim de caracterizar o perfil dos participantes, abordando, dessa forma, questões de múltipla escolha e discursivas. Foram registradas no questionário as dosagens de suplementação de vitamina $A$ recebidas, verificadas através do Cartão da Criança. RESULTADOS: Os resultados demonstraram baixos níveis de conhecimento acerca da vitamina A, bem como o PNVITA. COMCLUSÃO: Foi observado também que as doses administradas não estavam em conformidade com o que o programa estipula, ressaltando assim a importância do nutricionista na execução das ações voltadas à Alimentação e Nutrição, bem como de educação nutricional.
\end{abstract}

Palavras chave: Crianças. Hipovitaminose A. Saúde Pública. Vitamina A.

\footnotetext{
${ }^{1}$ Graduada em Nutrição pelo Centro Universitário Maurício de Nassau.

${ }^{2}$ Pós-graduanda em Nutrição Esportiva pelo Centro Universitário Unipê.

${ }^{3}$ Pós-graduando em Nutrição Clínica Funcional e Esportiva com Ênfase em Nutrigenômica pelo Centro Universitário Unifacisa.

${ }_{5}^{4}$ Pós-graduada em Nutrição Ortomolecular pela Faculdade IBRA.

${ }^{5}$ Especialista em Nutrição Clínica e Professora do curso de Nutrição pelo Centro Universitário Maurício de Nassau.
} 
ABSTRACT: The objective of this research was to evaluate the mother's and/or responsable perception of the National Vitamin A Supplementation Program. This is an exploratory, descriptive study of an applied nature. METHOD: The approach consisted of qualitative and quantitative research. The research was carried out in four health posts in the city of Campina Grande - PB. Data collection consisted of the application of a questionnaire about vitamin $A$ and the program containing the socioeconomic and demographic variables in order to characterize the profile of the participants, thus addressing multiple choice and discursive issues. The vitamin A supplementation dosages received, verified through the Children's Card, were recorded in the questionnaire. RESULTS: The results demonstrated low levels of knowledge about vitamin A as well as PNVITA. CONCLUSION: It was also observed that the administered doses were not in accordance with what the program stipulates, thus emphasizing the importance of the nutritionist in the execution of the actions directed to Food and Nutrition, as well as nutritional education.

Keywords: Children. Hypovitaminosis A. Public Health. Vitamin A 


\section{INTRODUÇÃO}

A vitamina A é um termo genérico que abrange um grupo de compostos químicos que apresentam biologicamente propriedades da vitamina. É uma vitamina de grande relevância, sendo, contudo, uma das mais estudadas (PHILIPPI; AQUINO, 2017). Apresenta-se sob a forma de retinol, retinal e ácido retinoico. Entretanto, nos mais variados tipos de tecidos suas funções são propiciadas à forma química ácido retinoico, com exceção da visão, cujo composto ativo existente é o retinal (RIBEIRO; MELO; TIRAPEGUI, 2018). É classificada como uma substância lipossolúvel, ou seja, necessita da ingestão de lipídeos na dieta para ser absorvido no trato gastrointestinal. Cerca de 50 a $80 \%$ da vitamina A é armazenada no fígado, sendo, portanto, sua reserva capaz de suprir as necessidades por vários meses (COZZOLINO, 2016). Com relação aos carotenoides, destacam-se o B-caroteno como sendo precursor importante da vitamina, sendo, por sua vez, convertido em vitamina A de acordo com a necessidade do organismo. É um micronutriente essencial, pois o organismo não é capaz de sintetizá-lo, fazendo-se necessário a ingestão por meio da dieta e/ou suplementação para desencadear as suas funções. Tem como função fisiológica mais conhecida e estabelecida no processo visual, sendo a cegueira noturna um dos primeiros sintomas de sua deficiência (SILVA; MURA, 2016). Além desta, desempenha funções básicas no organismo, tais como a manutenção da integridade dos tecidos epiteliais, a síntese de glicoproteínas, regulação da expressão gênica, produção de eritrócitos, síntese de testosterona, iniciação do impulso nervoso, sistema imunológico, atividade antioxidante, reprodução, além do crescimento e diferenciação celular (SILVA; MURA, 2016; COZZOLINO, 2016).

Embora o organismo não possa sintetizar a vitamina $A$, esta pode ser armazenada, onde será utilizada na medida de sua necessidade. Se há uma diminuição dessa reserva e, concomitantemente, não há uma ingestão adequada de fontes de vitamina A suficiente para atender as necessidades nutricionais do 
indivíduo, ocorre a deficiência (BRASIL, 2013). A deficiência de vitamina A (DVA) é uma das carências nutricionais que tem bastante repercussão na saúde da criança, trazendo consigo efeitos deletérios. Tem se tornado consideravelmente um problema de saúde pública, ficando abaixo apenas do ferro, onde atinge países subdesenvolvidos e em desenvolvimento, gerando agravos como retardo do crescimento e desenvolvimento, cegueira noturna, xeroftalmia, comprometimento do sistema imunológico, bem como desordens na produção de eritrócitos e reprodução (BRITO et al., 2016; MAHAN; RAYMOND, 2018). Tomando como base o relatório da Organização Mundial de Saúde, cerca de 190 milhões de crianças no mundo em idade pré-escolar têm concentração de retinol sérico abaixo de 0,70 $\mu \mathrm{mol} / \mathrm{l}$ (WHO, 2009). No Brasil, de acordo com os dados da última Pesquisa Nacional de Demografia e Saúde da Criança e da Mulher (PNDS) de 2006, em torno de 17,4\% das crianças menores de cinco anos e 12,3\% das mulheres apresentavam hipovitaminose $A$, sendo os maiores índices registrados na região Sudeste $(21,6 \%)$ e Nordeste (19\%) (BRASIL, 2009). Nesta última região, a Paraíba apresentou uma prevalência de DVA em crianças menores de cinco anos correspondendo a $21,8 \%$ (QUEIROZ, 2011). Em virtude da alta prevalência, em 13 de maio de 2005, através da Portaria 729 do Ministério da Saúde (MS), foi instituído o Programa Nacional de Suplementação de Vitamina A (PNVITA) que consiste na suplementação profilática com megadoses de vitamina A em crianças de 6-59 meses de idade com o objetivo de prevenir e controlar a DVA. De acordo com o MS, o profissional de saúde deverá verificar no cartão de vacinação da criança se as doses estão sendo administradas de forma correta, além de orientar a mãe e/ou responsável pela criança a respeito da importância dessa conduta, bem como a orientação adequada sobre alimentos fontes de vitamina A (BRASIL, 2013).

Sendo assim, este trabalho objetivou avaliar o conhecimento das mães acerca do Programa Nacional de Suplementação de Vitamina A. 


\section{METODOLOGIA}

Trata-se de um estudo exploratório, descritivo, de natureza aplicada. A abordagem foi constituída de pesquisa qualitativa e quantitativa, realizada em quatro Unidades Básicas de Saúde da Família (UBSF) do município de Campina Grande PB: UBS Beija Flor Zona Rural I, UBS Bem Te Vi Zona Rural II, Centro de Saúde do Catolé e UBS Adriana Bezerra Carvalho.

A seleção da amostra foi aleatória do tipo não probabilística, sendo composta por 100 mães e/ou responsáveis de crianças com idade entre 6 a 59 meses de idade, sendo 50 da zona urbana e 50 da zona rural que compareceram nas UBSF. Os critérios de inclusão foram mães e/ou responsáveis por crianças com idade superior a seis meses e inferior a cinco anos que aceitasse participar da pesquisa, mediante assinatura de Termo de Consentimento Livre e Esclarecido. A pesquisa não apresentou nenhum tipo de risco ao participante, tendo em vista que não manuseou com sangue, secreções ou materiais perfuro cortantes, sendo preservada sua imagem e identidade.

A coleta de dados foi realizada nos dias de atendimento as crianças nas UBSF e consistiu na aplicação de um questionário adaptado proposto por Queiroz (2011) contendo questões de múltipla escolha e discursivas. Este, constituiu de cinco questões abertas sobre a vitamina $A$, são elas: "1. O que você entende sobre vitamina A?", "2. O que a deficiência de vitamina A pode causar?", "3. Qual a importância/função da vitamina A para o organismo?", "4. Quais as fontes alimentares da vitamina A?", "5. Do que se trata o PNVITA?" e quatro questões fechadas, abordando sobre o aleitamento materno e o PNVITA. Para caracterização dos participantes, foram coletados dados com as variáveis socioeconômicas e demográficas. Foram registradas no questionário as dosagens de suplementação de vitamina $A$ recebidas, verificadas através do Cartão da Criança. De acordo com o Programa, as quantidades recomendadas de doses variam com a idade das crianças, com isso, para possibilitar a análise, subdividiu-se a relação de dose recomendada/tomada em quatro faixas, são elas: todas, para aquelas crianças que 
tomaram todas as doses recomendadas; $50 \%$, para aquelas crianças que tomaram mais da metade das doses recomendadas; $\leq 50 \%$, para aquelas crianças que tomaram até metade das doses recomendadas e nenhuma para aquelas crianças que não tomaram nenhuma dose das recomendadas.

Todos os dados foram registrados em forma de planilhas do Microsoft Excel (Versão 2010) para uma melhor visualização dos resultados obtidos. Feito isso, realizou-se a interpretação dos mesmos, chegando as seguintes classificações para o entendimento do público alvo sobre o tema da presente pesquisa: satisfatório, pouco satisfatório, não soube responder e resposta incorreta. Por se tratar de resultados qualitativos as variáveis assumem valores em categorias, classes ou rótulos. Estes, por sua vez foram representados por meio de gráficos de coluna.

O projeto foi aprovado pelo Comitê de Ética em Pesquisa da Universidade Estadual da Paraíba, sob protocolo $\mathrm{n}^{\circ}$ 69655417.1.0000.5187, e seguiu todas as recomendações da Resolução 466/12 do Conselho Nacional de Saúde para pesquisas envolvendo seres humanos no Brasil.

\section{RESULTADOS E DISCUSSÃO}

Do número total de participantes da pesquisa, 95 (95\%) são do sexo feminino e apenas 05 (5,0\%) do sexo masculino. Dentre as características sociodemográficas, a faixa etária mais prevalente foi de 30-34 anos, correspondendo a 27\%. Quando interrogados sobre a renda familiar, a maioria relatou possuir até um salário mínimo (SM), correspondendo a 71\% (Tabela 1). Com relação ao perfil de escolaridade, a maioria da zona rural relatou possuir ensino fundamental incompleto (36\%) e ensino médio completo (36\%), já na zona urbana a maioria relatou possuir ensino médio completo $(32 \%)$ e ensino superior completo (22\%). Com relação à profissão, na amostragem referente à zona urbana (50\%) predomina-se a "Dona do Lar", correspondendo a 40\%; já para a população da zona rural, predomina-se a profissão de "Agricultor", onde corresponde a $54 \%$ da amostra. 
Tabela 1 - Faixa etária e renda familiar da população total da pesquisa. Valores em percentual.

\begin{tabular}{lcccc}
\hline & \multicolumn{2}{c}{ Zona Urbana } & \multicolumn{2}{c}{ Zona Rural } \\
\hline Gênero & $\mathrm{N}$ & $\%$ & $\mathrm{~N}$ & $\%$ \\
$\mathrm{~F}$ & 45 & 90 & 50 & 100 \\
$\mathrm{M}$ & 5 & 10 & 0 & 0 \\
Faixa etária (anos) & 1 & 2 & 5 & 10 \\
$15-19$ & 9 & 18 & 14 & 28 \\
$20-24$ & 7 & 14 & 13 & 26 \\
$25-29$ & 14 & 28 & 13 & 26 \\
$30-34$ & 18 & 36 & 5 & 10 \\
$35-54$ & 1 & 2 & & \\
$>54$ & 25 & 50 & 46 & 92 \\
Renda familiar & 15 & 30 & 4 & 8 \\
Até 1 SM & 10 & 20 & 0 & 0 \\
2 a 3 SM & & & & \\
4 a 5 SM & 0 & 0 & 1 & 2 \\
Grau de escolaridade & 8 & 16 & 18 & 36 \\
Sem escolaridade & 2 & 4 & 3 & 6 \\
Fundamental incompleto & 7 & 14 & 9 & 18 \\
Fundamental completo & 16 & 32 & 18 & 36 \\
Médio incompleto & 6 & 12 & 0 & 0 \\
Médio completo & 11 & 22 & 1 & 2 \\
Superior incompleto & & & & \\
Superior completo & & & & \\
\hline
\end{tabular}

Fonte: Autores (2017).

A Tabela 2 apresenta a distribuição conjunta das frequências relativas (proporções) com relação ao total para o entendimento das mães sobre a vitamina $A$. Pode-se, então, observar que o nível de conhecimento classificado como satisfatório foi muito baixo, representando apenas $7 \%$ do total. Outros $39 \%$ demostraram ter pouco conhecimento e o restante ou não soube responder (36\%) ou respondeu de forma incorreta (18\%). 
Tabela 2 - Grau de conhecimento dos participantes quanto ao entendimento da vitamina A.

\section{Procedência}

\begin{tabular}{lccc} 
Grau de conhecimento & Zona urbana & Zona rural & Total \\
\hline Conhecimento Satisfatório & $2,0 \%$ & $5,0 \%$ & $7,0 \%$ \\
Pouco Conhecimento & $19,0 \%$ & $20,0 \%$ & $39,0 \%$ \\
Não Soube Responder & $19,0 \%$ & $17,0 \%$ & $36,0 \%$ \\
Resposta Incorreta & $10,0 \%$ & $8,0 \%$ & $18,0 \%$ \\
\hline
\end{tabular}

Fonte: Autores (2017).

É importante destacar que as mães, na grande maioria das vezes, referiam-se as cápsulas a uma vacina. $O$ pouco conhecimento sobre a vitamina $A$ também foi observado no estudo de Martins et al. (2007), onde 74,6\% dos indivíduos referiram não ter nenhum tipo de conhecimento acerca do micronutriente e que apenas 10,9\% referiu ter obtido alguma vez informação sobre a mesma. Ainda de acordo com o autor supracitado, foi demonstrado que 6,3\% dos responsáveis também associaram as cápsulas a uma vacina ou medicamento e que quase metade dos responsáveis $(47,6 \%)$ não receberam nenhuma informação acerca da administração da vitamina A. Fato também constatado por Marques, Teixeira e Paes (2017), que descreveram a vitamina A como um medicamento, gotinha ou vitamina de posto, relacionando a uma vitamina apenas quando perguntado sobre suas fontes e deficiência.

A segunda questão diz respeito ao grau de conhecimento das mães sobre a consequência da deficiência da vitamina $A$ para as crianças. De acordo com as respostas obtidas, $24 \%$ mostraram ter conhecimento satisfatório, sendo $15 \%$ desse valor referente às mães da zona urbana contra $9 \%$ das mães da zona rural. Destacase também o alto índice de mães que não souberam responder (55\%), sendo $31 \%$ da zona rural e $24 \%$ da zona urbana.

A Tabela 3 apresenta a distribuição conjunta das frequências relativas (proporções) com relação ao total das variáveis: grau de conhecimento e procedência das mães. 
Tabela 3 - Grau de conhecimento dos participantes quanto a deficiência da vitamina A.

\section{Procedência}

\begin{tabular}{lccc} 
Grau de conhecimento & Zona urbana & Zona rural & Total \\
\hline Conhecimento Satisfatório & $15,0 \%$ & $9,0 \%$ & $24,0 \%$ \\
Pouco Conhecimento & $2,0 \%$ & $3,0 \%$ & $5,0 \%$ \\
Não Soube Responder & $24,0 \%$ & $31,0 \%$ & $55,0 \%$ \\
Resposta Incorreta & $9,0 \%$ & $7,0 \%$ & $16,0 \%$ \\
\hline
\end{tabular}

Fonte: Autore (2017).

A Tabela 3 apresenta a distribuição conjunta das frequências relativas (proporções) com relação ao total das variáveis: grau de conhecimento e procedência das mães.

Tabela 3 - Grau de conhecimento dos participantes quanto a deficiência da vitamina A.

\section{Procedência}

\begin{tabular}{lccc} 
Grau de conhecimento & Zona urbana & Zona rural & Total \\
\hline Conhecimento Satisfatório & $15,0 \%$ & $9,0 \%$ & $24,0 \%$ \\
Pouco Conhecimento & $2,0 \%$ & $3,0 \%$ & $5,0 \%$ \\
Não Soube Responder & $24,0 \%$ & $31,0 \%$ & $55,0 \%$ \\
Resposta Incorreta & $9,0 \%$ & $7,0 \%$ & $16,0 \%$ \\
\hline
\end{tabular}

Fonte: Autores (2017)

Estudo recente publicado no Lancet Global Health estimou a prevalência da DVA entre 1991 e 2013 e sua carga de mortalidade em países de baixa e média renda indicou uma diminuição considerável na prevalência de DVA no leste e sudeste da Ásia e Oceania de $42 \%$ para 6\%, enquanto que na América Latina e Caribe foi de $21 \%$ para $11 \%$. Entretanto, em 2013, a prevalência da DVA mantevese elevada na África Subsaariana (48\%) e no sul da Ásia (44\%), apesar da carga de mortalidade em virtude desta carência nutricional ter sido reduzida em mais de 50\% desde 2000 (A STEVENS et al., 2015). A formação do hábito alimentar é iniciada ainda na infância, sendo consolidadas práticas que tendem a se repetir ao longo dos anos e, quando inadequados, poderão contribuir negativamente na saúde desses 
indivíduos quando adultos, as quais serão mais difíceis de modificá-las (OLIVEIRA; SAMPAIO; COSTA, 2014).

Quando foi perguntado às mães sobre as funções que a vitamina $A$ desempenham, a maioria não soube responder ou apresentou uma resposta incorreta, correspondendo a 76\%. Evidencia-se também a grande discrepância das mães que vivem na zona urbana sobre as da zona rural quanto ao grau de conhecimento satisfatório, sendo $16 \%$ da zona urbana e $1 \%$ na zona rural, como pode ser verificado na Tabela 4.

Tabela 4 - Grau de conhecimento dos participantes a respeito da função da vitamina A.

\section{Procedência}

\begin{tabular}{lccc} 
Grau de conhecimento & Zona urbana & Zona rural & Total \\
\hline Conhecimento Satisfatório & $16,0 \%$ & $1,0 \%$ & $17,0 \%$ \\
Pouco Conhecimento & $4,0 \%$ & $3,0 \%$ & $7,0 \%$ \\
Não Soube Responder & $21,0 \%$ & $27,0 \%$ & $48,0 \%$ \\
Resposta Incorreta & $9,0 \%$ & $19,0 \%$ & $28,0 \%$ \\
\hline
\end{tabular}

Fonte: Autores (2017).

Isso, provavelmente, pode ser justificado pelo fato das mães da zona urbana apresentarem maior nível de escolaridade, que está associada ainda as condições socioeconômicas da família. Segundo Almeida et al. (2010) e Reis e Crespo (2009), quanto maior a renda familiar, melhores serão as condições de saúde da criança e que o aumento dos anos de estudos é diretamente proporcional ao grau de conhecimento dos indivíduos.

Ao serem perguntadas sobre quais alimentos são fontes de vitamina $A, 41 \%$ das participantes responderam corretamente. As que foram classificadas com pouco conhecimento ou não souberam responder correspondem a 42\% (Tabela 5). 
Tabela 5 - Grau de conhecimento dos participantes a respeito das fontes alimentares da vitamina $\mathrm{A}$.

\section{Procedência}

\begin{tabular}{lccc} 
Grau de conhecimento & Zona urbana & Zona rural & Total \\
\hline Conhecimento Satisfatório & $19,0 \%$ & $22,0 \%$ & $41,0 \%$ \\
Pouco Conhecimento & $12,0 \%$ & $9,0 \%$ & $21,0 \%$ \\
Não Soube Responder & $12,0 \%$ & $9,0 \%$ & $21,0 \%$ \\
Resposta Incorreta & $7,0 \%$ & $10,0 \%$ & $17,0 \%$ \\
\hline
\end{tabular}

Fonte: Autores (2017).

Esperava-se que os responsáveis e, principalmente as mães, mencionassem o leite materno como fonte de vitamina $A$, contudo apenas $4 \%$ desse público citaram esse tipo de alimento. Foram relatados pelas mães frutas, verduras e hortaliças de forma generalizada como fonte da vitamina A. De acordo com Novaes et al. (2016), foi verificado que $75,8 \%$ das crianças tiveram um consumo adequado de vitamina $A$ em comparação ao proposto pelo Programa Nacional de Alimentação Escolar (PNAE), sendo que apenas 31,3\% tiveram um consumo adequado de lipídio. Fatores sociais, culturais, psicológicos, socioeconômicos e o modo como essas famílias vivem interferem diretamente na formação dos hábitos alimentares das crianças. Dessa forma, os pais e/ou responsáveis exercem papel crucial na formação destas práticas alimentares (BENTO; ESTEVES; FRANÇA, 2015). Observa-se no estudo de Oliveira (2015) uma inadequação do consumo da vitamina A em crianças entre 12 e 32 meses de idade, avaliado a partir da EAR (Estimated Average Requirement), uma prevalência de $19,8 \%$ nos meninos e $7,4 \%$ em meninas, correspondendo a $27,2 \%$. Fernandes (2015), ao avaliar a prática alimentar de crianças entre 06 a 23 meses de idade atendidas pela Estratégia Saúde da Família no Estado do Maranhão, verificou que houve um índice de inadequação de vitamina A de 76,3\% em crianças de 18 e 23 meses de idade, não atendendo às recomendações nutricionais para essa faixa etária. A baixa ingestão de alimentos fontes de vitamina $A$ ou a exclusão destes na alimentação estão correlacionados a hábitos alimentares, fatores socioeconômicos e aspectos culturais que afetam, sobretudo, na capacidade de escolha desses alimentos (QUEIROZ et al., 2013). 
Por fim, ao serem questionadas sobre o PNVITA, a grande maioria demonstrou não conhecer o programa, onde 39,1\% não souberam responder e $34,8 \%$ deram resposta incorreta, totalizando 73,9\%. A população da zona rural contribuiu significativamente para o valor apontado anteriormente. Apenas 17,4\% dos participantes apresentaram conhecimento satisfatório, como pode ser observado na Tabela 6.

Tabela 6 - Grau de conhecimento dos participantes sobre o PNVITA.

\begin{tabular}{lccc}
\hline Grau de conhecimento & $\begin{array}{c}\text { Procedência } \\
\text { Zona urbana }\end{array}$ & Zona rural & Total \\
\hline Conhecimento Satisfatório & $8,7 \%$ & $8,7 \%$ & $17,4 \%$ \\
Pouco Conhecimento & $8,7 \%$ & $0,0 \%$ & $8,7 \%$ \\
Não Soube Responder & $13,0 \%$ & $26,1 \%$ & $39,1 \%$ \\
Resposta Incorreta & $13,0 \%$ & $21,8 \%$ & $34,8 \%$ \\
\hline
\end{tabular}

Fonte: Autores (2017).

Mediante os resultados obtidos, foram constatados baixos níveis de conhecimento sobre o PNVITA. De acordo com os dados da Secretaria de Atenção Primária à Saúde (SAPS) em 2017, na Paraíba, o percentual de cobertura do Programa na faixa etária de 6-11 meses de idade esteve em $68,31 \%$, enquanto que na faixa etária de 12-59 meses, em sua segunda dose, essa percentagem caiu para $51,52 \%$. Além disso, a perda de cápsulas de vitamina A registrados por motivos de acondicionamento inadequado, extravio, perda na administração, prazo de validade e utilização indevida chegou a 1740 e 1728 para as cápsulas de 100mil UI e 200mil UI respectivamente (BRASIL, 2017). A falta de conhecimento do Programa tanto por parte dos profissionais de saúde quanto dos responsáveis pode repercutir na operacionalização do mesmo, mostrando assim relação direta à sua baixa cobertura (ALMEIDA et al., 2010).

A figura 1 apresenta a distribuição total da quantidade de doses tomadas pelas crianças nas zonas urbana e rural. Observa-se que na zona urbana (Figura 1a), $46 \%$ das crianças tomaram todas as doses recomendadas pelo programa, $22 \%$ tomaram mais da metade das doses, $28 \%$ tomaram menos da metade das doses e apenas $4 \%$ não tomaram nenhuma dose. Já para a população da zona rural (Figura 1b), um número menor de crianças tomou todas as doses (32\%) quando comparado 
a zona urbana, assim como destaca-se o alto índice de crianças que não tomaram nenhuma dose, correspondendo a 3 vezes mais, mesmo dado da zona urbana. No que se refere ao registro na caderneta de suplementação de vitamina $A$ nas doses preconizadas pelo MS, 91,7\% das crianças nos últimos 6 meses apresentavam o cartão de vacina desatualizado, mostrando desse modo uma baixa cobertura do programa (NOVAES et al., 2016). Jardim et al. (2019), avaliou a cobertura do PNVITA em crianças de 6 a 59 meses de idade no município de São Luís-MA, entre 2011 a 2016, cuja coleta de dados se deu por consulta na base de dados da Coordenação Geral de Alimentação e Nutrição em 2017, onde a cobertura oscilou entre $36,51 \%$ e $47,05 \%$, não atingindo, portanto, a meta proposta pelo Programa. Ainda de acordo com o autor supracitado, ao comparar o município com as demais capitais do Nordeste, foi observado que São Luís possui a menor média de cobertura dos 6 anos estudados (43,76\%), seguido da capital Recife $(48,11 \%)$, Maceió (49,04\%), Natal (49,79\%), Fortaleza (64,63\%), Aracajú (65\%), João Pessoa $(65,26 \%)$, Salvador $(66,3 \%)$ e Piauí $(68,51 \%)$.

Figura 1 - Distribuição total da quantidade de doses tomadas pelas crianças: (a) Zona urbana e (b) Zona rural.

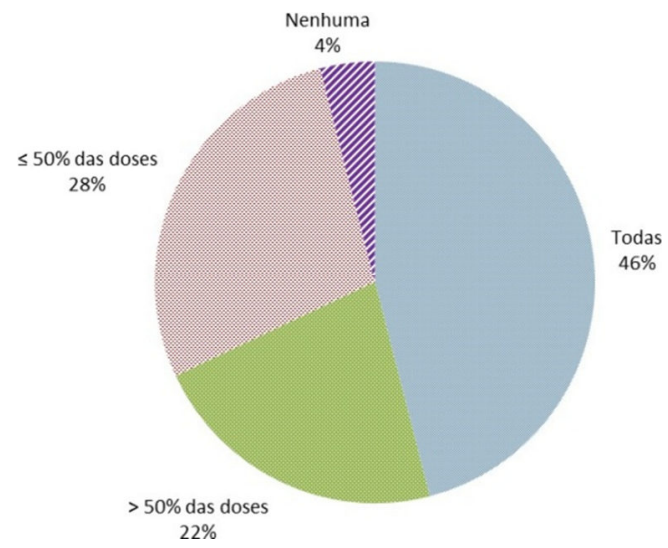

(a)

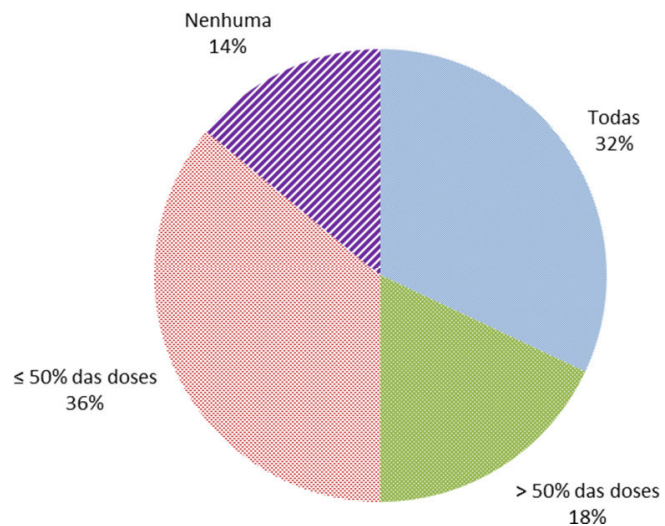

$18 \%$
Dividiu-se a escolaridade do conjunto de mães em sete níveis, são eles: sem escolaridade, ensino fundamental incompleto, ensino fundamental completo, ensino médio incompleto, ensino médio completo, ensino superior incompleto e ensino superior completo. Em seguida, observou-se como a escolaridade das mães pode influenciar no número de doses tomadas pelas crianças das zonas urbana e rural. As 
figuras 2 e 3 apresentam um gráfico de coluna relacionando as escolaridades com as quantidades de doses da suplementação para as zonas urbana e rural, respectivamente.

Inicialmente foi evidenciado que não houve a presença de pessoas sem escolaridade na população da zona urbana. Analisando aqueles com ensino médio completo, observa-se que apenas $37,5 \%$ atendem o total ideal de doses, outros $25 \%$ tomaram mais da metade do valor ideal, 37,5\% tomaram até a metade das doses e não foi evidenciado pessoas que não tenham tomado nenhuma dose para esta escolaridade. Tratando-se do maior nível, o superior completo, tem-se $63,6 \%$ que tomaram todas as doses, por outro lado, foi nessa escolaridade que houve o maior índice de pessoas que não tomaram nenhuma dose da suplementação $(18,2 \%)$. Notou-se ainda que no nível superior incompleto foi o que apresentou maior percentual de doses tomadas $(83,3 \%)$ e que $16,7 \%$ tomaram mais de $50 \%$ das doses, representadas na figura 2 .

Figura 2 - Quantidade de doses tomadas com relação às recomendadas pelo programa na zona urbana.

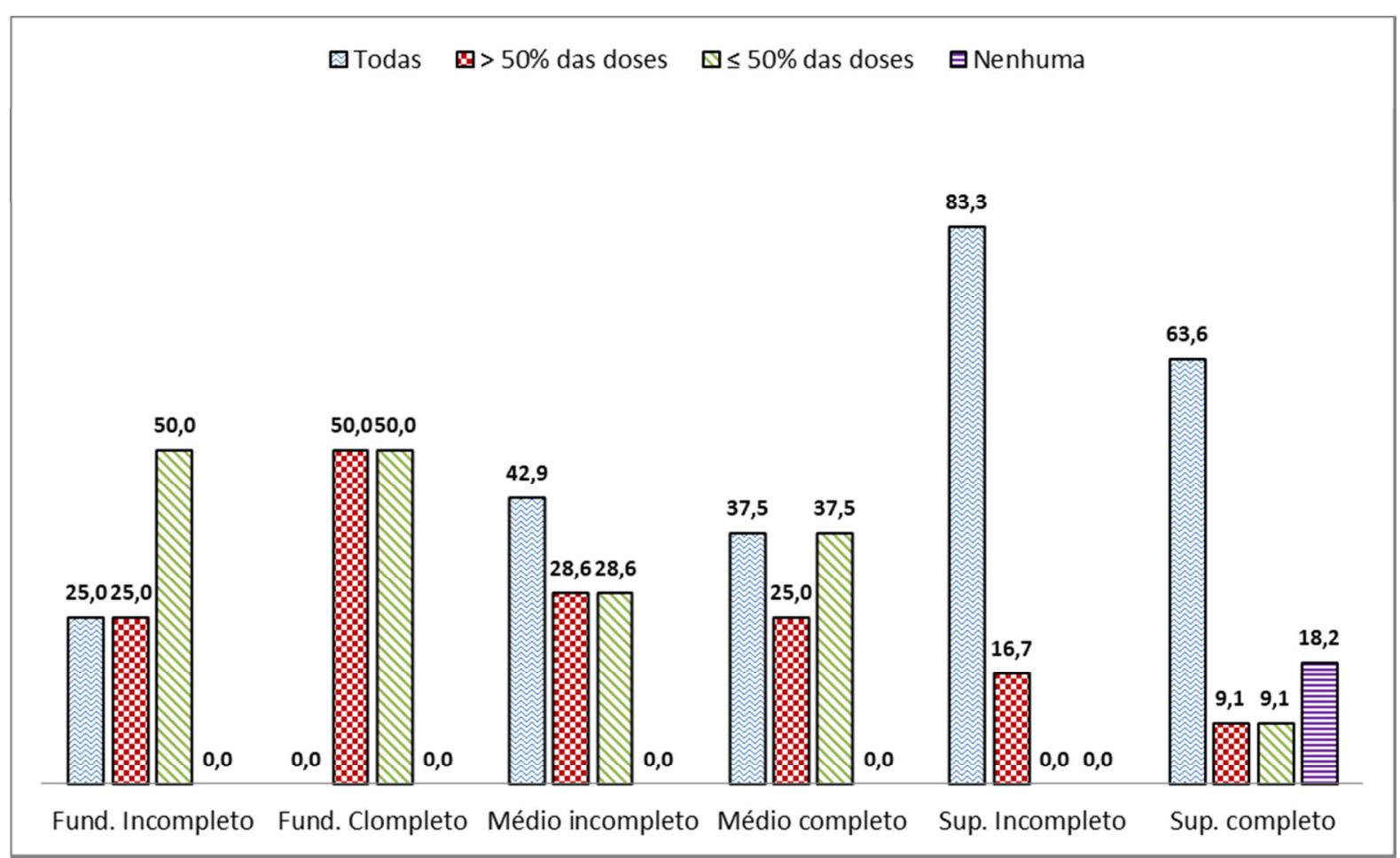

Revista Interdisciplinar em Saúde, Cajazeiras, 7 (1): 1678-1695, 2020, ISSN: 2358-7490. 
Tratando-se da zona rural, destaca-se positivamente que todos sem escolaridade tomaram mais da metade da quantidade recomendada e aqueles com escolaridade superior completo atendem plenamente $\mathrm{O}$ total de doses, diferentemente da zona urbana (Figura 2) que houve uma grande parcela que não tinha tomado nenhuma dose. As demais escolaridades apresentam predominância da quantidade de doses menor que a metade do recomendado pelo PNVITA.

Figura 3 - Quantidade de doses tomadas com relação às recomendadas pelo programa na zona rural.

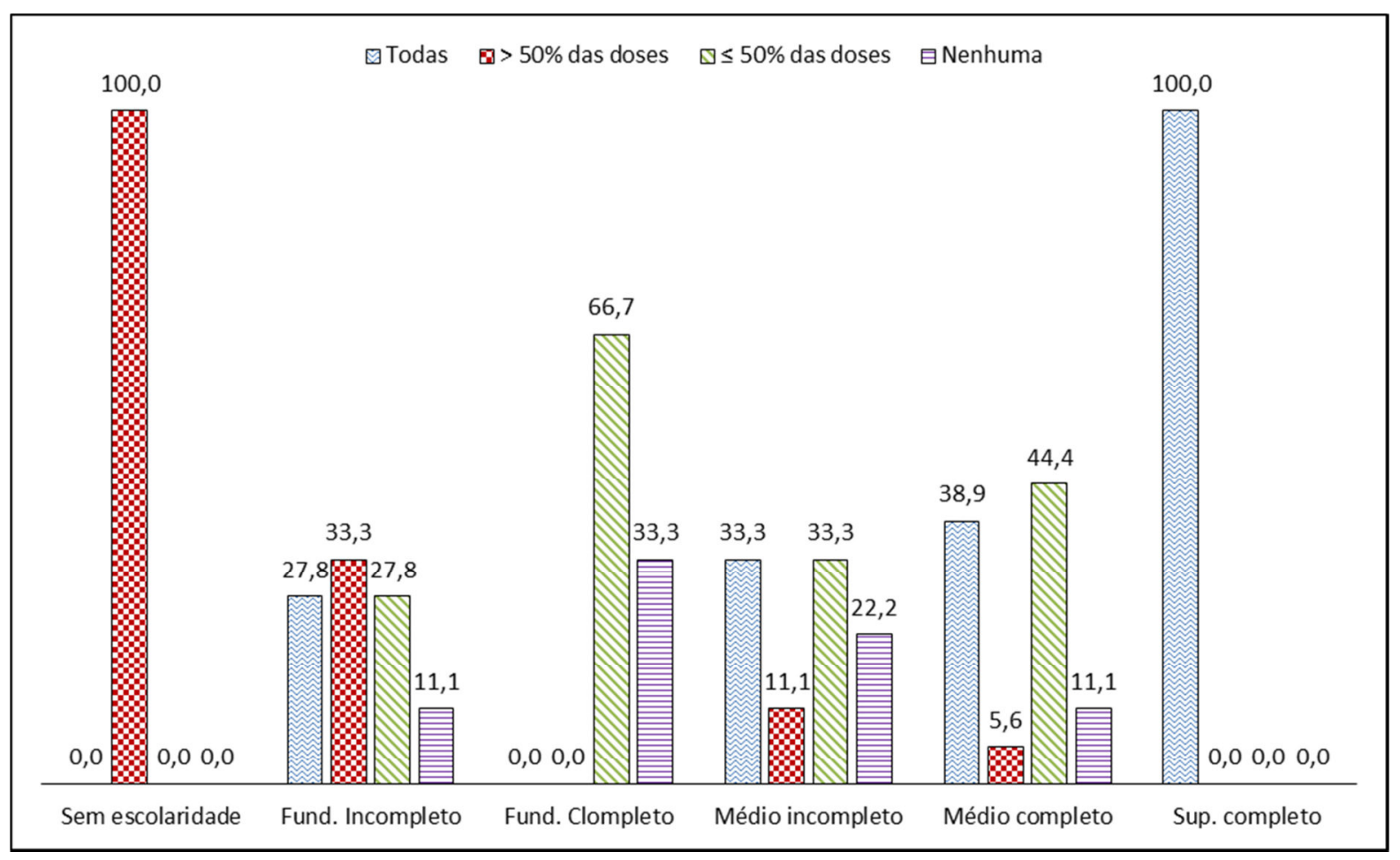

Sabe-se que o conhecimento inadequado influencia diretamente na atitude. Desta forma, se o conhecimento for insatisfatório, poderá comprometer a prática de todo o processo. Faz-se necessária uma atuação eficaz por parte de todos os profissionais de saúde, propondo estratégias educativas com os pais e/ou responsáveis pelas crianças, a fim de melhorar não só o conhecimento acerca dos alimentos pela população de baixa renda, como também ações que favoreçam a aquisição de atitudes e práticas adequadas, contribuindo assim para a prevenção das carências nutricionais na população infantil (SILVEIRA et al., 2014). Foi também 
observado percentuais maiores de cobertura do programa em crianças de 6 a 11 meses do que para crianças de 12 a 59 meses de idade, fato esse preocupante.

\section{CONCLUSÃO}

Apesar da hipovitaminose A demonstrar-se um importante problema de base nutricional, ainda são poucos os estudos que avaliam as respostas do Programa em referência aos atores envolvidos (mães, profissionais e gestores). Nesse contexto, fica clara a necessidade de serem desenvolvidas novas estratégias no PNVITA para estimular a educação nutricional de forma permanente, destacando assim a importância do nutricionista no âmbito da atenção primária à saúde para controle e combate a DVA, tendo em vista que, quando o indivíduo faz suas próprias escolhas a partir da reflexão do que the foi transmitido, ocorre uma mudança nos hábitos alimentares que farão parte de sua rotina. Além disto, quando este profissional não compõe a equipe multidisciplinar de saúde pode resultar em execução superficial das condutas relacionadas à Alimentação e Nutrição.

\section{REFERÊNCIAS BIBLIOGRÁFICAS}

A STEVENS, Gretchen et al. Trends and mortality effects of vitamin A deficiency in children in 138 low-income and middle-income countries between 1991 and 2013: a pooled analysis of population-based surveys. The Lancet Global Health, [s.I.], v. 3, n. 9, p.528-536, set. 2015. Elsevier BV. http://dx.doi.org/10.1016/s2214-109x(15)00039-x.

ALMEIDA, Erika Rodrigues de et al. Avaliação participativa do Programa Nacional de Suplementação de Vitamina A em um município da Região Nordeste do Brasil. Cadernos de Saúde Pública, [s.I.], v. 26, n. 5, p.949-960, maio 2010. FapUNIFESP (SciELO). http://dx.doi.org/10.1590/s0102-311x2010000500017.

BENTO, Isabel Cristina; ESTEVES, Juliana Maria de Melo; FRANÇA, Thaís Elias. Alimentação saudável e dificuldades para torná-la uma realidade: percepções de pais/responsáveis por préescolares de uma creche em Belo Horizonte/MG, Brasil. Ciência \& Saúde Coletiva, [s.l.], v. 20 , n. 8, p.2389-2400, ago. 2015. FapUNIFESP (SciELO). http://dx.doi.org/10.1590/141381232015208.16052014 .

BRASIL. Ministério da Saúde. Conselho Nacional de Saúde. Resolução n. 466/12. Diretrizes e normas regulamentadoras de pesquisa envolvendo seres humanos. Brasília: 2012. 
BRASIL. Ministério da Saúde. Pesquisa Nacional de Demografia e Saúde da Criança e da Mulher - PNDS 2006: dimensões do processo reprodutivo e da saúde da criança/ Ministério da Saúde, Centro Brasileiro de Análise e Planejamento. Brasília: Ministério da Saúde, 2009.

BRASIL. Ministério da Saúde. Secretaria de Atenção à Saúde. Departamento de Atenção Básica. Manual de condutas gerais do Programa Nacional de Suplementação de Vitamina A / Ministério da Saúde, Secretaria de Atenção à Saúde, Departamento de Atenção Básica. Brasília: Ministério da Saúde, 2013.

BRASIL. Ministério da Saúde. Secretaria de Atenção Primária à Saúde (SAPS). 2017. [acesso em $2020 \quad$ Mar 02]. Disponível em: <https://sisaps.saude.gov.br/micronutrientes/vitaminaa/relatorio>.

BRITO, Virginia Rossana de Sousa et al. Percepção de profissionais de saúde sobre o programa de combate à deficiência de vitamina A. Revista Brasileira em Promoção da Saúde, [s.l.], v. 29, n. 1, p.93-99, 30 mar. 2016. Fundacao Edson Queiroz. http://dx.doi.org/10.5020/18061230.

COZZOLINO, Silvia M. Franciscato. Biodisponibilidade de Nutrientes. 5. ed. São Paulo: Manole, 2016. $1430 \mathrm{p}$.

FERNANDES, Fabiana Corrêa. Prática alimentar de lactentes atendidos pela Estratégia Saúde da Família no Maranhão. 2015. 71 f. Dissertação (Mestrado) - Nutrição em Saúde Pública, Universidade de São Paulo (USP), São Paulo, 2015.

JARDIM, Ana Tamires et al. COBERTURA DA SUPLEMENTAÇÃO DE VITAMINA A EM CRIANÇAS DE 6 A 59 MESES NO MUNICÍPIO DE SÃO LUIS-MA. Revista UningÁ, [s. I.], v. 56, n. 3, p.51-59, set. 2019.

MAHAN, L. Kathleen; RAYMOND, Janice L. Krause: Alimentos, Nutrição e Dietoterapia. 14. ed. Rio de Janeiro: Elsevier, 2018. 1256 p.

MARQUES, Elisângela Christina Siqueira; TEIXEIRA, Romero Alves; PAES, Silvia Regina. THE NATIONAL VITAMIN A SUPPLEMENTATION PROGRAM: PRACTICES AND SOCIAL REPRESENTATIONS OF MOTHERS IN THE MUNICIPALITY OF VALE DO JEQUITINHONHA. Demetra: Alimentação, Nutrição \& Saúde, [s.I.], v. 12, n. 4, p.1165- 1183, 5 set. 2017. Universidade de Estado do Rio de Janeiro. http://dx.doi.org/10.12957/demetra.2017.28065.

MARTINS, Maisa Cruz et al. Avaliação de políticas públicas de segurança alimentar e combate à fome no período 1995-2002. 3 - o Programa Nacional de Controle da Deficiência de Vitamina A. Cadernos de Saúde Pública, [s.I.], v. 23, n. 9, p.2081-2093, set. 2007.

MARTINS, Maisa Cruz et al. Avaliação de políticas públicas de segurança alimentar e combate à fome no período 1995-2002. 3 - o Programa Nacional de Controle da Deficiência de Vitamina A. Cadernos de Saúde Pública, [s.I.], v. 23, n. 9, p.2081-2093, set. 2007. FapUNIFESP (SciELO). http://dx.doi.org/10.1590/s0102-311x2007000900016.

NOVAES, Taiane Gonçalves et al. Prevalence and factors associated to vitamin A deficiency in children attending public day care centers in the Southwest of Bahia. Revista Brasileira de Saúde Materno Infantil, [s.I.], v. 16, n. 3, p.337-344, set. 2016. FapUNIFESP (SciELO). http://dx.doi.org/10.1590/1806-93042016000300007.

OLIVEIRA, Bianca Rodrigues de. Prevalência de Inadequação do Consumo de Micronutrientes e Estado Nutricional de Crianças de 12 a 32 Meses de Idade, em São Luís, Maranhão. 2015. 64 f. TCC (Graduação) - Curso de Nutrição, Centro de Ciências Biológicas e da Saúde, Universidade Federal do Maranhão, São Luís, 2015. 
OLIVEIRA, Maria Nilka; SAMPAIO, Thereza Maria Tavares; COSTA, Eveline Alencar. EDUCAÇÃO NUTRICIONAL DE PRÉ-ESCOLARES: UM ESTUDO DE CASO. Oikos: Revista Brasileira de Economia Doméstica, Viçosa, v. 25, n. 1, p.93-113, 2014.

PHILIPPI, Sonia Tucunduva; AQUINO, Rita de Cássia de (Org.). Recomendações Nutricionais: Nos Estágios de Vida e Nas Doenças Crônicas Não Transmissíveis. São Paulo: Manole, 2017. 364 p.

QUEIROZ, Daiane de et al. Deficiencia de vitamina A e fatores associados em criancas de areas urbanas. Revista de Saúde Pública, [s.I.], v. 47, n. 2, p.248-256, jun. 2013. FapUNIFESP (SciELO). http://dx.doi.org/10.1590/s0034-8910.2013047002906.

QUEIROZ, Daiane de. Deficiência de vitamina A em crianças do estado da Paraíba, Brasil: perfil epidemiológico e associação com índices antropométricos. 2011. 71 f. Dissertação (Mestrado) - Centro de Ciências Biológicas e da Saúde, Universidade Estadual da Paraíba, Campina Grande, 2011.

REIS, Maurício; CRESPO, Anna. O IMPACTO DA RENDA DOMICILIAR SOBRE A SAÚDE INFANTIL NO BRASIL. Rio de Janeiro. p.7-22, fev. 2009.

RIBEIRO, Sandra Maria Lima; MELO, Camila Maria de; TIRAPEGUI, Julio. Avaliação Nutricional: Teoria e Prática. 2. ed. Rio de Janeiro: Guanabara Koogan, 2018. 340 p.

SILVA, Sandra M. Chemin S. da; MURA, Joana D'arc Pereira. Tratado de Alimentação, Nutrição e Dietoterapia. 3. ed. São Paulo: Payá, 2016. 1338 p.

SILVEIRA, Marcela Lima et al. Knowledge, attitude and practice on regional food among families of preschool children. Revista da Rede de Enfermagem do Nordeste, [s.I.], v. 15, n. 1, p.37-44, 16 fev. 2014. Rev Rene - Revista da Rede de Enfermagem de Nordeste. http://dx.doi.org/10.15253/2175-6783.2014000100006.

WHO. Global prevalence of vitamin A deficiency in populations at risk 1995-2005. WHO Global Database on Vitamin A Deficiency. Geneva, World Health Organization, 2009. 